\title{
Usefulness of dorsal laser evoked potentials in patients with spinal cord damage: report of two cases
}

\author{
GD Iannetti, A Truini, F Galeotti, A Romaniello, M Manfredi, G Cruccu
}

\begin{abstract}
Stimulation of the dorsal skin with brief laser impulses easily evokes brain potentials (laser evoked potentials, LEPs). Dorsal LEPs were first used to study the conduction velocity in the human spinothalamic tract. In this study the diagnostic usefulness of this technique was assessed by recording dorsal LEPs in two patients with focal spinal cord lesions (one intrinsic and the other extrinsic) and spared lemniscal sensitivities. In both cases, the brain evoked potentials were normal after stimulation of the metamers above the lesion but absent after stimulation of those below. Dorsal LEP recordings may prove a useful tool in localising lesions and in the neurophysiological assessment of focal spinal cord lesions involving the anterolateral quadrants of the spinal cord.
\end{abstract}

(F Neurol Neurosurg Psychiatry 2001;71:792-794)

Keywords: laser evoked potentials; spinothalamic tract; spinal cord lesions

Department of

Neurological Sciences,

University of Rome

"La Sapienza", Viale

Università 30, 00185

Roma, Italy

GD Iannetti

A Truini

F Galeotti

A Romaniello

M Manfredi

G Cruccu

Center for Sensory Motor Interaction, Aalborg, Denmark A Romaniello

Neuromed Institute, Pozzilli, Italy

M Manfredi

G Cruccu

Correspondence to: Dr G Cruccu

cruccu@uniroma1.it

Received 30 April 2001 and in revised form

20 July 2001

Accepted 6 August 2001 extrinsic). Both patients gave their informed consent and the research was approved by the local ethics committee. The level of their lesion had been precisely ascertained from the history and neuroimaging findings.

\section{Case reports}

PATIENT 1

Twelve years before presenting, this 71 year old man sustained a gunshot wound involving the spinal cord. Since the injury the patient had reported lower limb paraparesis and gradually diminishing pain and temperature sense from T12 to L1 level. Neurological examination failed to determine a clear sensory level. The patient therefore underwent neuroradiological and neurophysiological examinations. A CT scan of the spinal cord and $x$ ray films of the vertebral column (fig 1 A) disclosed a foreign body, the remains of a gun bullet within the spinal canal at the level of the T10 vertebral body. Recordings of somatosensory evoked potentials (SEPs) after electrical tibial nerve stimulation yielded small amplitude cortical signals at normal latency. For testing of dorsal LEPs we used a $\mathrm{CO}_{2}$ laser stimulator (Neurolas, Electronic Engineering, Florence, Italy) to generate brief pulses (wavelength $10.6 \mu \mathrm{m}$, beam diameter $2.5 \mathrm{~mm}$, stimulus intensity 1.5-15 W, duration 5-50 ms). The main negative $\left(\mathrm{N}_{2}\right)$ and positive $\left(\mathrm{P}_{2}\right)$ components of the LEPs were recorded from the vertex through a silver disc electrode (impedance below $5 \mathrm{k} \Omega$ ) referred to linked earlobes $\left(\mathrm{A}_{1} \mathrm{~A}_{2}\right)$, with simultaneous electro-oculographic recording. The technique used for recording dorsal LEPs is described in detail elsewhere. ${ }^{10}$ Laser stimuli were delivered to the skin overlying the vertebral spinous processes of $\mathrm{C} 5$ and the two thoracic vertebrae (T8 and T12) bordering the lesion rostrally and caudally. The perceptive threshold was larger after T12 than after C5 and T8 stimulation $\left(13.5 \mathrm{~mJ} / \mathrm{mm}^{2}\right.$ and 4.5 $\mathrm{mJ} / \mathrm{mm}^{2}$ ). Stimuli delivered to the two districts rostral to the lesion at about three times perceptive threshold intensity elicited easily recordable LEPs (C5: $\mathrm{N}_{2} 182 \mathrm{~ms}, \mathrm{P}_{2} 266 \mathrm{~ms}$, peak to peak amplitude $8 \mu \mathrm{V}$; T8: $\mathrm{N}_{2} 214 \mathrm{~ms}, \mathrm{P}_{2}$ $295 \mathrm{~ms}$, peak to peak amplitude $6 \mu \mathrm{V}$ ). Conversely, stimuli delivered to T12, even at a high intensity $\left(45 \mathrm{~mJ} / \mathrm{mm}^{2}\right)$, evoked no brain responses (fig $1 \mathrm{~A}$ ). 
PATIENT 2

A 70 year old woman had a history of spondylogenous myelopathy at the level of the C4-C5 vertebral bodies. In 1998, after a diagnosis of herniated disc $\mathrm{C} 4-\mathrm{C} 5$, the patient underwent a discectomy via the anterolateral approach (fig 1 B). When the patient presented for LEP testing she manifested clinical symptoms of spastic
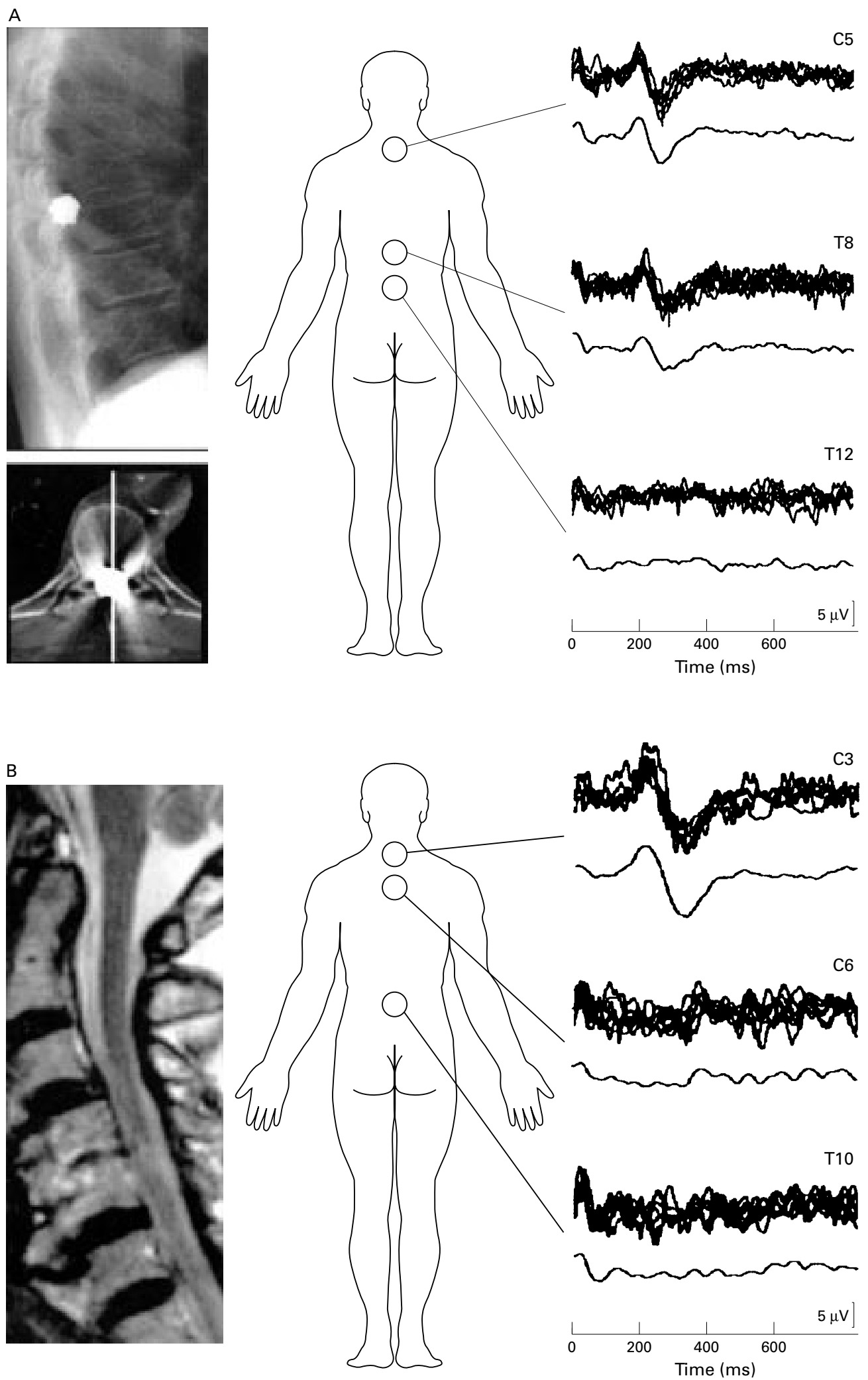

Figure 1 (A) Patient 1; CT and plain x ray film showing a foreign body (gun bullet) within the spinal cord, at the level of T10 (left panel). (B) Patient 2; MRI showing the results of the operation at the level of C4-C5 vertebral spinous processes. (A-B) right panels: laser stimulation of the rostral metamers at the level of the lesion (C5 and T8 in patient 1 and $C 3$ in patient 2) elicited wide amplitude, easily reproducible $\mathrm{N}_{2}-P_{2}$ evoked potentials in both patients whereas stimulation of the territories below the lesion (T12 in patient 1; C6 and T10 in patient 2) did not. 
paraparesis, neurologic bladder, and pain and temperature hypoaesthesia below the C5-C6 level. Lemniscal sensitivity was spared. The dorsal LEPs were recorded after stimulation of the skin overlying the vertebral spinous processes of a rostral vertebra (C3) and the two vertebrae (C6 and T10) caudal to the lesion. At the $\mathrm{C} 3$ level the perceptive threshold was 4.5 $\mathrm{mJ} / \mathrm{mm}^{2}$, and stimulation delivered at three times threshold intensity readily evoked a wide amplitude, reproducible brain potential $\left(\mathrm{N}_{2}\right.$ $230 \mathrm{~ms}, \mathrm{P}_{2} 340 \mathrm{~ms}$, peak to peak amplitude 16 $\mu \mathrm{V})$. At both the lower levels (C6, T10) the perceptive threshold was abnormally high and brain responses were absent, even in response to stimulation at high intensity $\left(45 \mathrm{~mJ} / \mathrm{mm}^{2}\right)$ (fig 1 B).

\section{Discussion}

In these two patients, both of whom had documented focal lesions of the spinal cord, intrinsic in one case (patient 1) and extrinsic in the other (patient 2), LEP recordings after dorsal stimulation (dorsal LEPs) $^{10}$ identified the precise level of the lesion.

Despite our patients' advanced age, the laser stimulation of the unaffected dermatomeres yielded large and easily reproducible dorsal LEPs, even in response to low intensity stimuli. In an earlier study ${ }^{10}$ we investigated a population younger than 34 years of age. The results we report here indicate that also in elderly persons dorsal LEPs can be easily recorded with no need to increase stimulus intensity.

In both patients we found markedly increased perceptive thresholds to laser stimulation in the hypaesthetic areas. Even high intensity laser pulses evoked no brain responses. The absence of brain potentials agreed with the clinically documented level of pain and temperature hypoaesthesia and with the neuroimaging findings. Hence we conclude that the technique of dorsal LEPs will identify the precise level of spinal cord lesions involving the anterolateral quadrant.

In the two patients we present here the findings from clinical examination, neuroimaging, and dorsal LEP recordings agreed. Diagnostic concordance is not, however, the rule. Many spinal cord lesions cause subtle clinical manifestations that make precise localisation of the level of lesion impossible. Another frequent drawback is the poor association between MRI findings and the functional deficit. Especially during the course of inflammatory disease, areas of abnormal MRI signal do not necessarily imply tissue damage or dysfunction. ${ }^{11}{ }^{12}$ Patients with overt clinical and neurophysiological signs may also have negative findings on MRI. In patients with clinical signs suggestive of spinothalamic system involvement and normal MRI findings, dorsal LEPs can be of great help in assessing the level of spinal cord dysfunction. Because anatomical studies show that before terminating on the neurons of the dorsal horn, the primary $\mathrm{A} \delta$ afferent fibres ascend some spinal segments in the Lissauer's tract, a key point is that the damaged myelomere always lies rostral to the dermatome where the LEP disappears.

Although both patients we studied had bilateral pain and temperature hypaesthesia, dorsal LEPs lend themselves equally well to the study of monolateral spinal lesions, characterised by unilateral involvement of the spinothalamic tract (for example, partial Brown-Séquard syndrome). In these cases stimulating the paravertebral skin at a distance of $3 \mathrm{~cm}$ from the midline will ensure that the ipsilateral spinothalamic tract makes no contribution to the brain evoked potential.

Others have assessed the level of spinal cord lesions by delivering laser or electrical pulses to the limbs. ${ }^{4671314}$ A disadvantage of techniques using limb stimulation is that they allow only a qualitative, non-localising diagnosis of the presence or absence of a spinal cord lesion. They can also be strongly influenced by possible changes in peripheral afferent conduction. Recording of dorsal LEPs, conversely, offers a dual advantage. Firstly, it minimises the peripheral component of the LEP, thus directly exploring the central thermal pain pathways; secondly, it allows stimulation at all levels along the spinal cord, thus localising the lesion with precision.

In conclusion, although this technique has to be tested prospectively, dorsal LEPs seem to provide a simple, inexpensive, and dependable investigative tool for the assessment of focal lesions within the spinal cord.

1 Bromm B, Treede RD. Nerve fibre discharges, cerebral potentials and sensations induced by $\mathrm{CO}_{2}$ laser stimulation. Hum Neurobiol 1984;3:33-40.

2 Bromm B, Chen AC. Brain electrical source analysis of laser evoked potentials in response to painful trigeminal nerve stimulation. Electroencephalogr Clin Neurophysiol 1995;95: $14-26$.

3 Valeriani $M$, Rambaud L, Mauguière F. Scalp topography and dipolar source modelling of potentials evoked by $\mathrm{CO}_{2}$ laser stimulation of the hand. Electoencephalogr Clin Neurophysiol 1996;100:343-53.

4 Treede RD, Lankers J, Frieling A, et al. Cerebral potentials evoked by painful laser stimuli in patients with syringomyelia. Brain 1991;114:1595-607.

5 Arendt-Nielsen L. Characteristics, detection, and modulation of laser-evoked potentials. Acta Anaesthesiol Scand tion of laser-evoked poten

6 Kakigi R, Shibasaki H, Kuroda Y, et al. Pain-related somatosensory evoked potentials in syringomyelia. Brain 1991; 14:1871-89

7 Bromm B, Frieling A, Lankers J. Laser evoked potentials in the assessment of disturbed pain and temperature sensibility. Electroencephalogr Clin Neurophysiol 1991;80:284-91.

8 Kakigi R, Kuroda Y, Neshige R, et al. Physiological study of the spinothalamic tract conduction in multiple sclerosis. $\mathcal{F}$ Neurol Sci 1992;107:205-9.

9 Cruccu G, Romaniello A, Amantini A, et al. Assessment of trigeminal small-fiber function: brain and reflex responses evoked by $\mathrm{CO}_{2}$-laser stimulation. Muscle Nerve 1999;22: 508-16.

10 Cruccu G, Iannetti GD, Agostino R, et al. Conduction velocity of the human spinothalamic tract as assessed by velocity of the human spinothalamic tract as assessed
laser evoked potentials. Neuroreport 2000;11:3029-32.

11 Turano G, Jones SJ, Miller DH, et al. Correlation of SEPs abnormalities with brain and cervical cord MRI in multiple sclerosis. Brain 1991;114:663-81.

12 Comi G, Filippi M, Rovaris M, et al. Clinical, neurophysiological, and magnetic resonance imaging correlations in multiple sclerosis. F Neurol Neurosurg Psychiatry 1998;64: S21-5.

13 Lorenz J, Hansen HC, Kunze K, et al. Sensory deficits of a nerve root lesion can be objectively documented by somatosensory evoked potentials elicited by painful infrared laser stimulations: a case study. F Neurol Neurosurg Psychiatry 1996;61:107-10.

14 Restuccia D, Insola A, Valeriani M, et al. Somatosensory evoked potentials after multisegmental lower limb stimulation in focal lesions of the lumbosacral spinal cord. $\mathcal{F}$ Neurol Neurosurg Psychiatry 2000;69:91-5. 